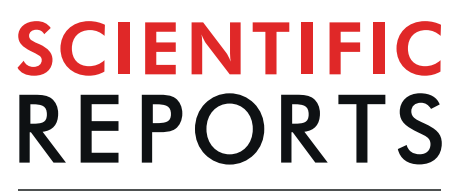

natureresearch

Check for updates

\title{
Correctors modify the bicarbonate permeability of F508del-CFTR
}

\author{
Michele Fiore ${ }^{\bowtie}$, Cristiana Picco \& Oscar Moran
}

One of the most common mutations in Cystic Fibrosis (CF) patients is the deletion of the amino acid phenylalanine at position 508. This mutation causes both the protein trafficking defect and an early degradation. Over time, small molecules, called correctors, capable of increasing the amount of mutated channel in the plasma membrane and causing an increase in its transport activity have been developed. This study shows that incubating in vitro cells permanently transfected with the mutated channel with the correctors VX809, VX661 and Corr4a, and the combination of VX809 and Corr4a, a recovery of anion transport activity is observed. Interestingly, the permeability of bicarbonate increases in the cells containing corrected p.F508del CFTR channels is greater than the increase of the halide permeability. These different increases of the permeability of bicarbonate and halides are consistent with the concept that the structural conformation of the pore of the corrector-rescued p.F508del channels would be different than the normal wild type CFTR protein.

Cystic fibrosis (CF), caused by mutation in Cystic Fibrosis Transmembrane Regulator (CFTR) gene ${ }^{1,2}$, is one of the most common autosomal recessive lethal inherited diseases in the Caucasian poulation ${ }^{3,4}$. The CFTR protein is an ATP-binding cassette transporter with two membrane-spanning domains (MSD1 and MSD2), two nucleotide binding domains (NBD1 and NBD2) and a unique regulatory domain (R) ${ }^{5}$. The protein works as an ATP- and phosphorylation-regulated anion channel ${ }^{6}$, that is permeable to the halides $\left(\mathrm{Br}^{-}, \mathrm{Cl}^{-}, \mathrm{I}^{-}, \mathrm{F}^{-}\right)^{7,8}$, and can also conduct $\mathrm{HCO}_{3}^{-}$with a ratio of the permeability between bicarbonate and chloride $\left(P_{\mathrm{HCO} 3} / P_{\mathrm{Cl}}\right)$ of $\sim 0.25^{9-11}$. The physiological role of CFTR is the maintenance of fluid transport across epithelial cells of airways, intestines, pancreatic as well as bile ducts ${ }^{12}$. The CF respiratory disease is the main cause of death and the main determinant of the burden on the quality of life. Most of the health problems in CF respiratory disease can be attributed to the viscous mucus phenotype, hindering ciliary activity and their clearance mechanism ${ }^{13}$. This pathogenicity is caused by the malfunctioning of the CFTR channel which does not adequately transport chloride and bicarbonate across the plasma membrane causing lack of ions and water homeostasis at the surface of the airway epithelia ${ }^{14,15}$.

To date, more than 2,000 mutations have been identified that cause CF, classified in six classes according to the effect of the mutation leading to anion transport defects in epithelia ${ }^{16}$. The most common mutation is the deletion of phenylalanine 508, which is located in the NBD1 (p.F508del, class II mutation), causing aberrant assembly of the full-length protein and making the channel susceptible to premature degradation via the protein quality control mechanism ${ }^{16,17}$. The p.F508del-CFTR protein is a functional anion channel, but it is unstable and rapidly degrading, leading to a great reduction of chloride and bicarbonate transport by the CFTR channel ${ }^{18-20}$.

In the CF patients, the reduction of bicarbonate transport gives rise to a lower $\mathrm{pH}$ in airways surface liquid $(\mathrm{ASL})^{15,21}$ and interferes with the ASL fluidity. The ASL is composed of mucus and periciliary liquid layer (PLC) and is very important to keep intact the defense mechanisms of the respiratory airway. Low concentration of $\mathrm{HCO}_{3}^{-}$may interfere with the post-secretory modifications of the mucins MUC5AC and MUC5B ${ }^{15,22}$. When the mucus retains the physiological characteristics, the cilia of the airways epithelium are able to discharge the mucus containing potential pathogens captured during breathing 22 .

As a feasible therapy for CF patients carrying the mutation p.F508del, small molecules, named correctors, that are able to increase the amount of protein in the plasma membrane have been developed ${ }^{23,24}$. It has been proposed that the molecules capable of correcting the mutated protein can be divided into three classes. Class 1 is represented by molecules that are likely to interact with the NBD1; class 2 is represented by molecules that would interact with the NBD2; class 3 correctors would facilitate the folding of the NBD1 segment or prevents it from being denatured ${ }^{25-27}$.

Here we examine the halide and bicarbonate permeability of the p.F508del-CFTR rescued by three correctors, VX809 and VX661, that belong to the class 1, and Corr4a, of class 2. We show that the rescued p.F508del-CFTR 
has a higher bicarbonate relative permeability than the wild type control. These results have important implications in the correctors-based CF therapeutic strategy.

\section{Methods}

Cell cultures. Fisher Rat Thyroid (FRT) cells stably transfected with a wild type (WT) CFTR, or CFTR carrying the cystic fibrosis mutation p.F508del, were grown at $37^{\circ} \mathrm{C}$ and $5 \% \mathrm{CO}_{2}$ in modified $\mathrm{F} 12$ Coon's medium with addition of $10 \% \mathrm{FBS}$ and, $2 \mathrm{mM}$ of Glutamine, $1 \mathrm{mg} \mathrm{ml}^{-1}$ penicillin, $100 \mu \mathrm{g} \mathrm{ml}^{-1}$ streptomycin and the addition of $1 \mathrm{mg} \mathrm{ml}^{-1}$ geneticin (G418) and $0.6 \mathrm{mg} \mathrm{ml}^{-1}$ zeocin as selection agents. For the iodide influx assay, cells stably co-transfected with the halide-sensitive yellow fluorescent protein, YFP-p.H148Q/p.I152L ${ }^{28,29}$, were seeded in 96-well microplates at a density of 40,000 cells/well. For intracellular pH measurements, cells were seeded on glass-bottom Petri dishes. Measurements were carried out $48 \mathrm{~h}$ after seeding. To evaluate the correctors p.F508del rescue on the cells expressing the mutated CFTR, cells were incubated for $18 \mathrm{~h}$ with $5 \mu \mathrm{M}$ of VX809 (lumacaftor; Selleck Chemicals, Huston, TX, USA), VX661 (tezacaftor; Selleck Chemicals), Corr4a (ChemBridge Corp. San Diego, CA, USA), or $2.5 \mu \mathrm{M}$ of VX809 with $2.5 \mu \mathrm{M}$ of Corr4a together (final concentration $5 \mu \mathrm{M})$. Except when indicated, all chemicals compounds were purchased from Sigma-Aldrich (Milan, Italy).

lodide Influx assay. To evaluate the halide transport activity of the CFTR channels, cells were incubated for 30 minutes with $20 \mu \mathrm{M}$ forskolin at $37^{\circ} \mathrm{C}$ in a solution containing (in $\mathrm{mM}$ ): $\left.\mathrm{KNO}_{3} 4.5, \mathrm{Ca}_{\left(\mathrm{NO}_{3}\right.}\right)_{2} 1.2, \mathrm{MgSO}_{4} 0.2$, Glucose 5, HEPES 20, pH 7.4 NaCl 136, in a final volume of $60 \mu \mathrm{L}$. Thus, we measured the influx of iodide, which causes the quenching of the YFP as enters into the cell. The fluorescence of YFP was monitored using a fluorescence plate reader (Tristar2 S, Berthold Technologies, Bad Wildbad, Germany), equipped with $485 \mathrm{~nm}$ excitation and $535 \mathrm{~nm}$ emission filters ${ }^{30,31}$.

After recording the fluorescence for 5 seconds as a baseline, cells are perfused with $100 \mu \mathrm{L}$ of a solution where $\mathrm{NaCl}$ was substituted by $\mathrm{NaI}$, and fluorescence was monitored every 0.2 seconds for a further 30 seconds. In this way, the final concentration of $\mathrm{NaI}$ in the bath was $85 \mathrm{mM}$. Measurements were performed at $37^{\circ} \mathrm{C}$.

The fluorescence time course was normalized by the average of the fluorescence of the baseline recorded before $\mathrm{NaI}$ injection. The initial rate of fluorescence decay $(\mathrm{QR})$ was derived by fitting the signal with a double exponential function. The QR is an indicator of halide transport by the CFTR 28,31 .

Bicarbonate transport in cells. The transport of bicarbonate was evaluated by measuring the variation of the intracellular $\mathrm{pH}\left(\mathrm{pH}_{\mathrm{i}}\right)$ following the $\mathrm{NH}_{4}{ }^{+}$prepulse technique ${ }^{31,32}$. The $\mathrm{pH}_{\mathrm{i}}$ was measured in FRT cells using the fluorescent $\mathrm{pH}$ indicator 2',7'-bis-2-(carboxyethyl)-5-(and6) carboxyfluorescein ester (BCECF-AM; Thermo Fisher Scientific, Waltham, MA USA). Cells were loaded with $5 \mu \mathrm{M}$ of BCECF-AM in the culture medium without serum for 30-40 min at room temperature. After loading, cells were washed two times with the recording solution containing (mM): $\mathrm{NaCl} 140, \mathrm{~K}_{2} \mathrm{HPO}_{4} 2.5, \mathrm{MgSO}_{4} 1, \mathrm{CaCl}_{2} 1$, HEPES 10, glucose $6(\mathrm{pH} 7.3)$ and were allowed to recover for at least $30 \mathrm{~min}$ before measurement. The Petri dish was mounted in a perfusion system in the stage of an epifluorescence inverted microscope (iMIC) with a Qimaging Retiga EXI Blue camera (Till Photonics, Graefelfing, Germany). During the experiments, the perfusion solutions were equilibrated with $5 \%$ $\mathrm{CO} 2$ and $95 \%$ air.

Cells were visualized with an Objective Plan Super Apochromat 10x (Olympus, Tokyo, Japan; N.A. 0.4, W. D. $30.1 \mathrm{~mm}$ ). For excitation, we used Till Oligochrome (FEI, Munich, Germany), a wavelength switching device containing a stable xenon light source. The sample was excited at two wavelengths, $440 \mathrm{~nm}$ and $490 \mathrm{~nm}$, and emission was recorded at $520 \mathrm{~nm}$. To calibrate the $\mathrm{pH}_{\mathrm{i}}$ measurements, the $\mathrm{pH}_{\mathrm{i}}$ was varied incubating the cells in a high potassium concentration solution with $15 \mu \mathrm{M}$ nigericin to equilibrate the intracellular compartment with various external $\mathrm{pH}$ values; a calibration curve was constructed plotting the $\mathrm{pH}$ against the ratio of fluorescence emitted upon excitation at the two excitation wavelengths ${ }^{31}$.

The $\mathrm{NH}_{4}{ }^{+}$solution was prepared by replacing $30 \mathrm{mM} \mathrm{NaCl}$ in the standard recording solution with an equimolar concentration of $\mathrm{NH}_{4} \mathrm{Cl}$. When cells were subjected to an acid load by the transient application (2-3 min pulse) of a $30 \mathrm{mM} \mathrm{NH}_{4}{ }^{+}$solution, the $\mathrm{pH}_{\mathrm{i}}$ rose as $\mathrm{NH}_{4}{ }^{+}$accumulated in the intracellular space during the $\mathrm{NH}_{4} \mathrm{Cl}$ perfusion. Cells were subsequently returned to a recording solution without $\mathrm{NH}_{4}^{+}$, and acidification of the cytoplasm occurred when $\mathrm{NH}_{3}$ quickly diffused out of the cell. For the bicarbonate recording solution, $30 \mathrm{mM}$ of $\mathrm{NaCl}$ was substituted by $\mathrm{NaHCO}_{3}$. The $\mathrm{Na}^{+} / \mathrm{H}^{+}$exchange and the $\mathrm{Na}^{+} / \mathrm{HCO}_{3}{ }^{-}$co-transport were inhibited with $1 \mathrm{mM}$ amiloride, and the $\mathrm{Cl}^{-} / \mathrm{HCO}_{3}{ }^{-}$and the $\mathrm{Na}^{+}$-dependent $\mathrm{Cl}^{-} / \mathrm{HCO}_{3}{ }^{-}$exchangers inhibited by adding $300 \mu \mathrm{M}$ disodium $4,4^{\prime}$-diisothiocyano-2,2'-stilbenedisulfonate (DIDS) to the recording solution ${ }^{33-35}$. Moreover, DIDS also inhibits the calcium-activated chloride channels bestrophin and anoctamin-1 that both display $\mathrm{HCO}_{3}{ }^{-}$permeability $^{36}$. CFTR was activated by the addition of $100 \mu \mathrm{M}$ of the permeable cAMP analog pCPT-cAMP to the recording solutions. The $\mathrm{HCO}_{3}{ }^{-}$influx was calculated from the $\mathrm{pH}_{\mathrm{i}}$, applying the Henderson-Hasselbach equation and assuming a constant $\mathrm{CO}_{2}$ partial pressure. Details of the $\mathrm{HCO}_{3}{ }^{-}$flux calculations are presented in the supplementary file.

Data analysis. The time course of the recorded fluorescence traces, as well as the statistical analysis, was evaluated using IgorPro (version 8.03, Wavemetrics, Portland, Oregon). Results are presented as mean \pm standard error (SEM) and the number of measurements. Statistical analysis was performed using Student's t-test or one-way ANOVA to compare the different data sets; $P$ values $<0.05$ were considered statistically significant.

\section{Results}

The iodide influx was measured as the initial quenching rate of the fluorescence, QR, in FRT cells expressing halide-sensitive YFP and WT-CFTR or the mutant p.F508del-CFTR, respectively. Application of $20 \mu \mathrm{M}$ of forskolin elicited the fluorescence decay after the addition of iodide to the external solution $\left(\mathrm{QR}=62.9 \pm 1.4 \mathrm{~ms}^{-1}\right.$; $\mathrm{n}=10$ ), implying iodide influx driven by the activation of the WT-CFTR channels (Fig. 1A). Similar experiments 
A

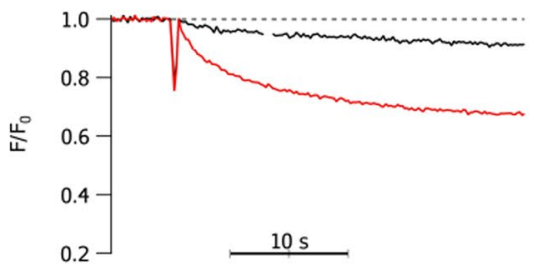

C

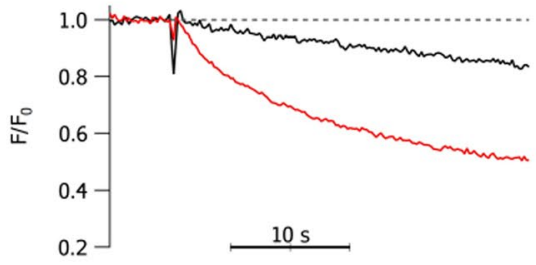

$\mathrm{E}$

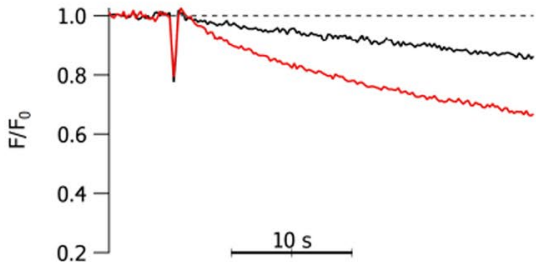

B

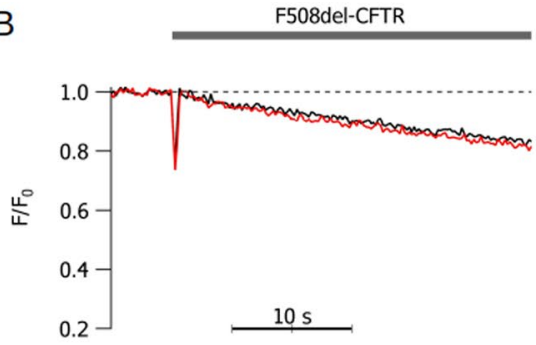

D

F508del-CFTR + VX661

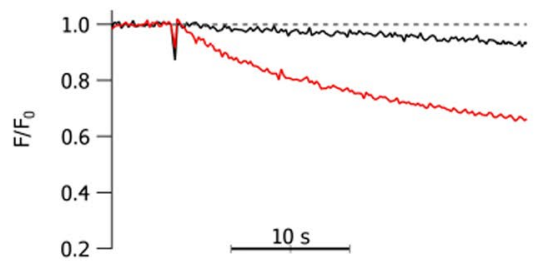

$\mathrm{F}$

F508del-CFTR + Vx809 + Corr4a

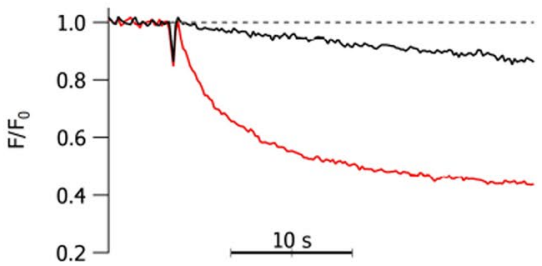

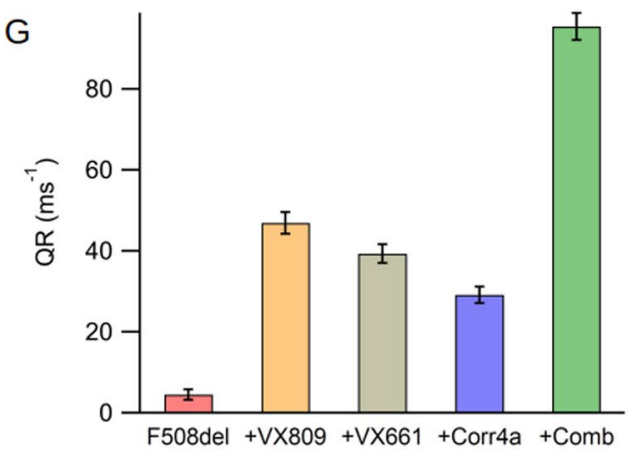

Figure 1. Iodide influx assay in FRT cells. Time course of the iodide-sensitive YFP fluorescence decay, normalized by the initial fluorescence, after the addition of $85 \mathrm{mM}$ of $\mathrm{NaI}$ in the external solution of WT-CFTR cells (A) and p.F508del-CFTR cells (B-F) before (black traces) and upon the addition of $20 \mu \mathrm{M}$ of forskolin to activate the channels (red trace). Panels (B-F) represent respectively the cells not incubated, incubated with VX809, VX661, Corr4a and the combination of VX809 and Corr4a together. The number of measures for each condition was between 9 and 12 . The $\mathbf{G}$ panel is the representation of the summary of the experiment that shows the change produced by each corrector. Data are the average $+/-$ SEM. The number of the experiment in each condition was between 9 and 12 .

were carried out on cells expressing p.F508del-CFTR, where the application of $20 \mu \mathrm{M}$ of forskolin elicited a very small iodide transport $\left(\mathrm{QR}=4.6 \pm 0.8 \mathrm{~ms}^{-1} ; \mathrm{n}=12\right)$. Incubation of the p.F508del-CFTR cells with $5 \mu \mathrm{M}$ of VX809, VX661, or Corr4a results a significant increase of the iodide influx elicited by forskolin, yielding a QR of $47.0 \pm 2.6 \mathrm{~ms}^{-1}(\mathrm{n}=12), 39.4 \pm 2.3 \mathrm{~ms}^{-1}(\mathrm{n}=9)$ and $29.2 \pm 1.4 \mathrm{~ms}^{-1}(\mathrm{n}=12)$, for VX809, VX661 and Corr4a, respectively (Fig. 1B-E). The treatment of the cells with a combination of $2.5 \mu \mathrm{M}$ VX809 and $2.5 \mu \mathrm{M}$ Corr4a results in a further increase of the iodide transport, yielding a QR of $95.5 \pm 2.9 \mathrm{~ms}^{-1}(\mathrm{n}=12)$, according to previous studies in which the combination of the two correctors has a synergistic activity (Fig. 1F) $)^{25-27,37}$. The graph in Fig. $1 \mathrm{G}$ shows a summary of all the QR values of the iodide influx experiments. 
A
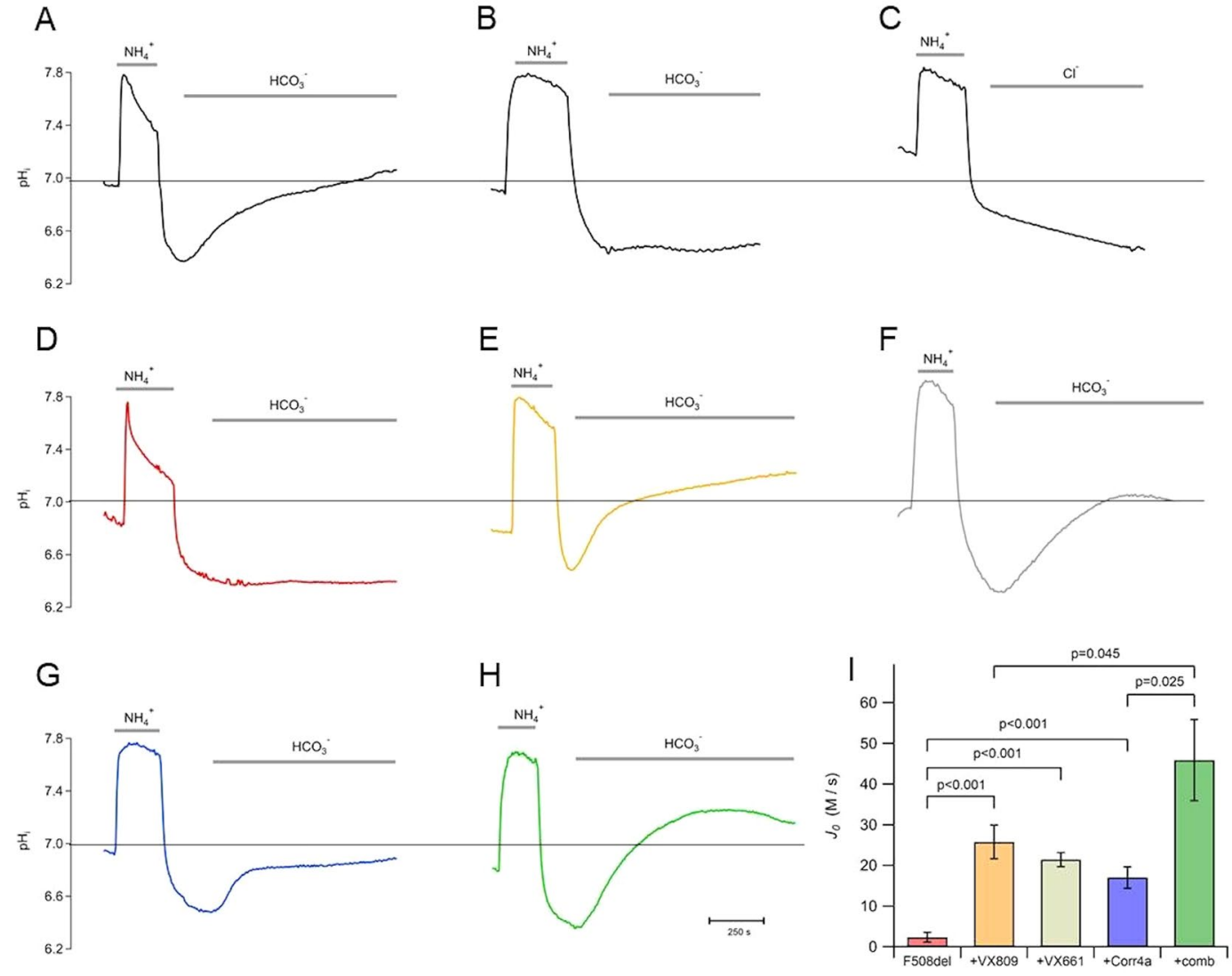

Figure 2. Intracellular $\mathrm{pH}$ measurement using ammonium pulse protocol (see methods). Perfusion of FRT

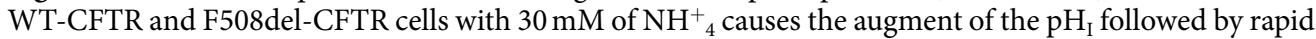
acidification when $\mathrm{NH}_{4}^{+}$is removed. When bicarbonate was perfused an alkalinization was observed upon the $100 \mu \mathrm{M}$ pCPT-CAMP stimulation in FRT WT-CFTR (A); FRT WT-CFTR without pCPT-CAMP stimulation or with $\mathrm{Cl}^{-}$in place of $\mathrm{HCO}_{3}{ }^{-}$was used as control (B,C). FRT p.F508del-CFTR cells not incubated with correctors do not elicit any $\mathrm{pH}_{\mathrm{i}}$ variation and consequent bicarbonate transport (D), while incubation with VX809, VX661, Corr4a and the combination of VX809 and Corr4a, gives rise to an $\mathrm{HCO}_{3}{ }^{-}$influx (E-H). The panel I show the summary of bicarbonate influx of p.F508del-CFTR not incubated (red); incubated with VX809 (orange), VX661 (gray), Corr4a (blue) and the combination (green) respectively. Data are means \pm SEM. The number of experiments for each condition was between 8 and 12 .

The transport of bicarbonate through the CFTR channels was evaluated measuring the intracellular $\mathrm{pH}$ response to the $\mathrm{NH}_{4}{ }^{+}$pulse protocol ${ }^{31,32}$. The washout of $\mathrm{NH}_{4}{ }^{+}$from the solution induces acidification of the cells; the restore of neutral $\mathrm{pH}_{\mathrm{i}}$ is observed when $\mathrm{HCO}_{3}{ }^{-}$is added to the bath and WT-CFTR is activated by pCPT-cAMP (Fig. 2A). The addition of DIDS and amiloride to the perfusion solutions assures that other $\mathrm{HCO}_{3}{ }^{-}$pathways are blocked, and therefore the intracellular alkalinization is due to the entrance of $\mathrm{HCO}_{3}{ }^{-}$ through the activated CFTR. Indeed, the alkalinization of the cells is not present when the CFTR was not activated by the cyclic nucleotide (Fig. 2B). Similarly, when the cells were perfused with an $\mathrm{HCO}_{3}{ }^{-}$free solution, even if the CFTR was activated by pCPT-cAMP, no $\mathrm{pH}_{\mathrm{i}}$ variation was observed (Fig. $2 \mathrm{C}$ ). These results show that the intracellular alkalinization of the cells expressing WT-CFTR is due to the $\mathrm{HCO}^{-}$influx through the CFTR channels.

When the experiments were carried out in cells expressing p.F508del-CFTR, in presence of $\mathrm{HCO}_{3}^{-}$, the activation of the mutant CFTR by pCPT-cAMP elicited a very small $\mathrm{pH}_{\mathrm{i}}$ variation after the $\mathrm{NH}_{4}{ }^{+}$pulse, that corresponds to an $\mathrm{HCO}_{3}{ }^{-}$influx of $2.4 \pm 1.2 \mu \mathrm{M} / \mathrm{s}(\mathrm{n}=10)$ (Fig. 2D). Conversely, the treatment with correctors rescues the p.F508del-CFTR activity, responding to the post-pulse $\mathrm{HCO}_{3}{ }^{-}$perfusion with an intracellular alkalinization, yielding an $\mathrm{HCO}_{3}{ }^{-}$influx of $25.8 \pm 4.1 \mu \mathrm{M} / \mathrm{s}(\mathrm{n}=12), 21.5 \pm 1.7 \mu \mathrm{M} / \mathrm{s}(\mathrm{n}=9)$ and $17.0 \pm 2.6 \mu \mathrm{M} / \mathrm{s}(\mathrm{n}=10)$ for the cells treated with VX809, VX661 and Corr4a, respectively (Fig. 2E-G). Similarly, as the data obtained measuring the halide transport, when cells expressing p.F508del-CFTR are treated with a mixture of the two correctors (VX809+ Corr4a), a faster alkalinization is observed consistent with an augmented $\mathrm{HCO}_{3}{ }^{-}$influx, $45.9 \pm 10.0 \mu \mathrm{M} / \mathrm{s}(\mathrm{n}=8)($ Fig. $2 \mathrm{H})$. These data confirm the synergistic effect of VX809 and Corr4a observed also in the iodide influx experiments. A summary of the quantitative evaluation of the $\mathrm{HCO}_{3}{ }^{-}$influx is shown in Fig. 2I.

Tables with the experimental data and the statistical comparisons are shown in the supplementary file. 


\section{Discussion}

The main purpose of this study was to observe the pharmacological effect of correctors, individually or combined, on the p.F508del-CFTR channel permeability. Hence, we evaluated the halide and the $\mathrm{HCO}_{3}{ }^{-}$transport driven by the p.F508del-CFTR channels rescued by three different correctors, VX809, VX661 and Corr4a. To avoid possible interferences of potentiators with the correctors-rescue of $\mathrm{CFTR}^{38,39}$, we have not applied any potentiator during the functional assay. The halide transport was done by measuring the kinetics of the fluorescence quenching of iodide-sensitive YFP. This method has been proven to yield data that are proportional to the $\mathrm{Cl}^{-}$transport capacity of the CFTR 29,40 . Thus, we observed that FRT cells permanently transfected with halide sensitive YFP and p.F508del-CFTR showed a very small iodide transport evoked by forskolin. Indeed as previously demonstrated, the p.F508del-CFTR mutation gives rise to a functional channel with a wrong conformation, that brings to rapid degradation, but only a small amount of channels reach the membrane ${ }^{18-20}$. The incubation of these cells with VX809, VX661 or Corr4a rescues of the mutated proteins and a greater expression of the channel on the membrane surface ${ }^{22,41-43}$, resulting in a significant increase of iodide transport by the rescued p.F508del-CFTR. Interestingly, while the incubation with VX809, VX661 or Corr4a yielded a 10.3, 8.6 and 6.4-fold increase of halide transport, respectively, the use of VX809 and Corr4a together produced a significantly larger 20.8 fold-increase, indicating a synergistic effect of the two correctors. These results are similar to those recently reported elsewhere ${ }^{43}$.

Since it is not possible to directly evaluate the transport of bicarbonate, we used the ammonium pulse technique $^{31,32}$. We first demonstrated that, in our experimental conditions, the post-pulse alkalinization of the cytoplasm requires the activation of the CFTR and the presence of extracellular $\mathrm{HCO}_{3}{ }^{-}$. Hence, the $\mathrm{HCO}_{3}{ }^{-}$influx in corrector-rescued p.F508del-CFTR cells was calculated from the post-pulse alkalinization rate. For the p.F508del-CFTR, the increase of the $\mathrm{HCO}_{3}{ }^{-}$influx is similar to that observed for the halide transport, yielding 10.9, 9.1, 7.2 and 19.5-fold increase for VX809, VX661, Corr4a, and the combination of VX809 and Corr4a, respectively. The linear correlation test of the $\mathrm{HCO}_{3}{ }^{-}$influx and halide transport data of the rescued p.F508del-CFTR yields a significant correlation $(r=0.89, P<0.0004)$. It indicates a similar ratio between the $\mathrm{HCO}_{3}{ }^{-}$influx and halide transport measured in the corrector-rescued p.F508del-CFTR of $0.54 \pm 0.02$.

The FRT cells co-expressing the iodide-sensitive YFP protein were obtained from the clones previously transfected with the WT- or F508del-CFTR genes. As a result, we can assume that the protein levels of CFTR genes between YFP and no YFP cell lines are identical. However, because the WT- and F508del-CFTR transfected cell lines are two different clones, the number of plasmid copies cannot be considered identical a priori. Consequently, it is not possible to compare the absolute values of the functional assays of ion transport between these CFTR isoforms with our preparation. However, independently from the absolute values, the $\mathrm{HCO}_{3}{ }^{-}$influx and halide transport ratio will reflect the $\mathrm{HCO}_{3}^{-}$/halide permeability ratios. Interestingly, the WT-CFTR has an $\mathrm{HCO}_{3}{ }^{-}$ influx and halide transport ratio of 0.23 , which is significantly smaller than that of the corrector treated p.F508del-CFTR $(P<0.0008)$. The augmented $\mathrm{HCO}_{3}{ }^{-}$influx and halide transport ratio of the corrector-rescued mutant-CFTR can be explained with a decrease of the halide permeability, or the increase of the $\mathrm{HCO}_{3}^{-}$permeability. We discard a reduction of the halide permeability by correctors treatment since different reports have indicated that the absolute halide permeability of rescued p.F508del channels is similar to that of the WT-CFTR ${ }^{44-46}$. Therefore, most probably there is an increase of the $\mathrm{HCO}_{3}{ }^{-}$permeability in the rescued mutants. This result for the VX809 is similar to that previously reported ${ }^{11}$; for the other two correctors, VX661 and Corr4a, this is the first report regarding the permeability of the rescued mutant-CFTR.

When human bronchial epithelia from CF-patients with the mutation p.F508del are treated with VX809 there is a reduction of the mucus viscosity, but there is not a significant effect in the fluid re-absorption by the epithe$\mathrm{lia}^{47}$. Since the homeostasis of the ionic content of the ASL is regulated by the CFTR-secretion of $\mathrm{Cl}^{-}$, the lack of fluid reabsorption can be explained by a reduced $\mathrm{Cl}^{-}$secretion due to an incomplete mutant rescue. However, the increased $\mathrm{HCO}_{3}{ }^{-}$permeability of the rescued p.F508del-CFTR would favor the $\mathrm{HCO}_{3}{ }^{-}$secretion, facilitating the post-secretional modification of the mucin, and the consequent reduction of the viscosity.

In summary, we showed that correctors of class 1, VX809 and VX661, a corrector of class 2 Corr4a, or a combination of the two classes of correctors, increase both halide and $\mathrm{HCO}_{3}{ }^{-}$transport on cells transfected with the p.F508del-CFTR channels. However, the rescued p.F508del-CFTR has a bigger relative bicarbonate permeability, independently to the corrector class. Thus, we expect that a combination of two class 1 correctors, nowadays used for CF treatment ${ }^{50}$, will not modify this higher bicarbonate permeability pattern. A difference in the ions permeability would reflect a different structure of the ion pore in the channel. Structural studies have revealed differences between WT and mutated proteins ${ }^{48,49}$. Paradoxically, this incomplete rescue of the p.F508del-CFTR may represent a therapeutical advantage, as the enhanced $\mathrm{HCO}_{3}{ }^{-}$secretion would favor the mucus fluidification.

The encouraging results obtained in this work show the need to use cell lines that endogenously express CFTR, both wild type and mutated, such as a bronchial cell line able to form epithelium. In this way it will be possible to clarify whether the effect of the correctors on the mutated protein observed in our experiments is due to the experimental conditions or whether the differentiation and polarization of the epithelium may play a role in modifying the properties of the CFTR rescued channels.

Received: 20 February 2020; Accepted: 29 April 2020;

Published online: 21 May 2020

\section{References}

1. Gadsby, D. C., Vergani, P. \& Csanády, L. The ABC protein turned chloride channel whose failure causes cystic fibrosis. Nature 440, 477-483 (2006)

2. Molinski, S. et al. Functional Rescue of F508del-CFTR Using Small Molecule Correctors. Front. Pharmacol. 3 (2012).

3. Bobadilla, J. L., Macek, M., Fine, J. P. \& Farrell, P. M. Cystic fibrosis: A worldwide analysis of CFTR mutations-correlation with incidence data and application to screening. Hum. Mutat. 19, 575-606 (2002).

4. Strausbaugh, S. D. \& Davis, P. B. Cystic Fibrosis: A Review of Epidemiology and Pathobiology. Clin. Chest Med. 28, 279-288 (2007). 
5. Cant, N., Pollock, N. \& Ford, R. C. CFTR structure and cystic fibrosis. Int. J. Biochem. Cell Biol. 52, 15-25 (2014).

6. Liu, F., Zhang, Z., Csanády, L., Gadsby, D. C. \& Chen, J. Molecular Structure of the Human CFTR Ion Channel. Cell 169, 85-95.e8 (2017).

7. Linsdell, P. Anion conductance selectivity mechanism of the CFTR chloride channel. Biochim. Biophys. Acta BBA - Biomembr 1858, 740-747 (2016).

8. Hwang, T.-C. \& Kirk, K. L. The CFTR ion channel: gating, regulation, and anion permeation. Cold Spring Harb. Perspect. Med 3, a009498 (2013).

9. Poulsen, J. H., Fischer, H., Illek, B. \& Machen, T. E. Bicarbonate conductance and pH regulatory capability of cystic fibrosis transmembrane conductance regulator. Proc. Natl. Acad. Sci. 91, 5340-5344 (1994).

10. Tang, L., Fatehi, M. \& Linsdell, P. Mechanism of direct bicarbonate transport by the CFTR anion channel. J. Cyst. Fibros. 8, 115-121 (2009).

11. Ferrera, L., Baroni, D. \& Moran, O. Lumacaftor-rescued F508del-CFTR has a modified bicarbonate permeability. J. Cyst. Fibros. 18, 602-605 (2019).

12. Saint-Criq, V. \& Gray, M. A. Role of CFTR in epithelial physiology. Cell. Mol. Life Sci. 74, 93-115 (2017).

13. Tang, A. C. et al. Current concepts: host-pathogen interactions in cystic fibrosis airways disease. Eur. Respir. Rev. 23, 320-332 (2014).

14. Frizzell, R. A. Ten Years With CFTR. Physiol. Rev. 79, S1-S2 (1999).

15. Quinton, P. M. Cystic fibrosis: impaired bicarbonate secretion and mucoviscidosis. The Lancet 372, 415-417 (2008).

16. Castellani, C. \& Assael, B. M. Cystic fibrosis: a clinical view. Cell. Mol. Life Sci. 74, 129-140 (2017).

17. Veit, G. et al. Structure-guided combination therapy to potently improve the function of mutant CFTRs. Nat. Med 24, 1732 (2018).

18. Drumm, M. L. et al. Chloride conductance expressed by delta F508 and other mutant CFTRs in Xenopus oocytes. Science 254, $1797-1799$ (1991).

19. Lukacs, G. L. et al. The delta F508 mutation decreases the stability of cystic fibrosis transmembrane conductance regulator in the plasma membrane. Determination of functional half-lives on transfected cells. J. Biol. Chem. 268, 21592-21598 (1993).

20. Shah, V. S. et al. Relationships among CFTR expression, HCO3 - secretion, and host defense may inform gene- and cell-based cystic fibrosis therapies. Proc. Natl. Acad. Sci. 113, 5382-5387 (2016).

21. Quinton, P. M. Physiological Basis of Cystic Fibrosis: A Historical Perspective. Physiol. Rev. 79, S3-S22 (1999).

22. Yang, N., Garcia, M. A. S. \& Quinton, P. M. Normal mucus formation requires cAMP-dependent HCO3- secretion and $\mathrm{Ca}^{2+}$ mediated mucin exocytosis: cAMP-dependent HCO 3 - secretion potentiates mucus discharge. J. Physiol. 591, 4581-4593 (2013).

23. Verkman, A. S. \& Galietta, L. J. V. Chloride channels as drug targets. Nat. Rev. Drug Discov. 8, 153-171 (2009).

24. Zegarra-Moran, O. \& Galietta, L. J. V. CFTR pharmacology. Cell. Mol. Life Sci. CMLS 74, 117-128 (2017).

25. Okiyoneda, T. et al. Mechanism-based corrector combination restores $\Delta$ F508-CFTR folding and function. Nat. Chem. Biol. 9 (2013).

26. Veit, G. et al. From CFTR biology toward combinatorial pharmacotherapy: expanded classification of cystic fibrosis mutations. Mol. Biol. Cell 27, 424-433 (2016).

27. Laselva, O., Molinski, S., Casavola, V. \& Bear, C. E. Correctors of the Major Cystic Fibrosis Mutant Interact through MembraneSpanning Domains. Mol. Pharmacol. 93, 612-618 (2018).

28. Galietta, L. J. V., Haggie, P. M. \& Verkman, A. S. Green fluorescent protein-based halide indicators with improved chloride and iodide affinities. FEBS Lett 499, 220-224 (2001).

29. Galietta, L. V. J., Jayaraman, S. \& Verkman, A. S. Cell-based assay for high-throughput quantitative screening of CFTR chloride transport agonists. Am. J. Physiol.-Cell Physiol 281, C1734-C1742 (2001).

30. Cossu, C. et al. Anion-Transport Mechanism of a Triazole-Bearing Derivative of Prodigiosine: A Candidate for Cystic Fibrosis Therapy. Front. Pharmacol. 9 (2018).

31. Fiore, M. et al. Small molecule-facilitated anion transporters in cells for a novel therapeutic approach to cystic fibrosis. Br. J. Pharmacol. 176, 1764-1779 (2019).

32. Kintner, D. B. et al. Increased tolerance to oxygen and glucose deprivation in astrocytes from $\mathrm{Na}^{+} / \mathrm{H}^{+}$exchanger isoform 1 null mice. Am. J. Physiol.-Cell Physiol 287, C12-C21 (2004).

33. Burnham, C. E., Amlal, H., Wang, Z., Shull, G. E. \& Soleimani, M. Cloning and Functional Expression of a Human Kidney $\mathrm{Na}^{+}: \mathrm{HCO}_{3}^{-}$Cotransporter. J. Biol. Chem. 272, 19111-19114 (1997).

34. Shumaker, H. \& Soleimani, M. CFTR upregulates the expression of the basolateral $\mathrm{Na}^{+}-\mathrm{K}^{+}-2 \mathrm{Cl}^{-}$cotransporter in cultured pancreatic duct cells. Am. J. Physiol.-Cell Physiol 277, C1100-C1110 (1999).

35. Lee, M. G. et al. Cystic Fibrosis Transmembrane Conductance Regulator Regulates Luminal $\mathrm{Cl}^{-} / \mathrm{HCO}_{3}{ }^{-}$Exchange in Mouse Submandibular and Pancreatic Ducts. J. Biol. Chem. 274, 14670-14677 (1999).

36. Hartzell, C. et al. Looking chloride channels straight in the eye: bestrophins, lipofuscinosis, and retinal degeneration. Physiol. Bethesda Md 20, 292-302 (2005).

37. Molinski, S. V. et al. Orkambi ${ }^{\circledR}$ and amplifier co-therapy improves function from a rare CFTR mutation in gene-edited cells and patient tissue. EMBO Mol. Med. 9, 1224-1243 (2017).

38. Cholon, D. M. et al. Potentiator ivacaftor abrogates pharmacological correction of $\Delta$ F508 CFTR in cystic fibrosis. Sci. Transl. Med. 6, 246ra96-246ra96 (2014).

39. Veit, G. et al. Some gating potentiators, including VX-770, diminish $\Delta$ F508-CFTR functional expression. Sci. Transl. Med. 6, 246ra97-246ra97 (2014).

40. Moran, O. \& Zegarra-Moran, O. On the measurement of the functional properties of the CFTR. J. Cyst. Fibros. 7, 483-494 (2008).

41. Pedemonte, N. et al. Small-molecule correctors of defective DeltaF508-CFTR cellular processing identified by high-throughput screening. J. Clin. Invest. 115, 2564-2571 (2005).

42. Van Goor, F. et al. Correction of the F508del-CFTR protein processing defect in vitro by the investigational drug VX-809. Proc. Natl. Acad. Sci. U. S. A. 108, 18843-18848 (2011).

43. Amico, G., Brandas, C., Moran, O. \& Baroni, D. Unravelling the Regions of Mutant F508del-CFTR More Susceptible to the Action of Four Cystic Fibrosis Correctors. Int. J. Mol. Sci. 20 (2019).

44. Dalemans, W. et al. Altered chloride ion channel kinetics associated with the $\Delta$ F508 cystic fibrosis mutation. Nature 354, 526-528 (1991).

45. Miki, H., Zhou, Z., Li, M., Hwang, T.-C. \& Bompadre, S. G. Potentiation of disease-associated cystic fibrosis transmembrane conductance regulator mutants by hydrolyzable ATP analogs. J. Biol. Chem. 285, 19967-19975 (2010).

46. de Wilde, G. et al. Identification of GLPG/ABBV-2737, a Novel Class of Corrector, Which Exerts Functional Synergy With Other CFTR Modulators. Front. Pharmacol 10, 514 (2019).

47. Gianotti, A. et al. Pharmacological rescue of mutant CFTR protein improves the viscoelastic properties of CF mucus. J. Cyst. Fibros. Off. J. Eur. Cyst. Fibros. Soc. 15, 295-301 (2016).

48. Baroni, D., Zegarra-Moran, O. \& Moran, O. Functional and pharmacological induced structural changes of the cystic fibrosis transmembrane conductance regulator in the membrane solved using SAXS. Cell. Mol. Life Sci. 72, 1363-1375 (2015).

49. Pollock, N. L., Satriano, L., Zegarra-Moran, O., Ford, R. C. \& Moran, O. Structure of wild type and mutant F508del CFTR: A smallangle X-ray scattering study of the protein-detergent complexes. J. Struct. Biol. 194, 102-111 (2016).

50. Lommatzsch, S. T. \& Taylor-Cousar, J. L. The combination of tezacaftor and ivacaftor in the treatment of patients with cystic fibrosis: clinical evidence and future prospects in cystic fibrosis therapy. Ther Adv Respir Dis 13, 1753466619844424 (2019). 


\section{Author contributions}

M.F. and O.M. planned the study. M.F. and C.P. performed the experiments. M.F. and O.M. wrote the manuscript.

\section{Competing interests}

The authors declare that the research was concluded in the absence of any commercial or financial relationship that could be construed as a potential conflict of interest.

\section{Additional information}

Supplementary information is available for this paper at https://doi.org/10.1038/s41598-020-65287-4.

Correspondence and requests for materials should be addressed to M.F.

Reprints and permissions information is available at www.nature.com/reprints.

Publisher's note Springer Nature remains neutral with regard to jurisdictional claims in published maps and institutional affiliations.

(c) (i) Open Access This article is licensed under a Creative Commons Attribution 4.0 International License, which permits use, sharing, adaptation, distribution and reproduction in any medium or format, as long as you give appropriate credit to the original author(s) and the source, provide a link to the Creative Commons license, and indicate if changes were made. The images or other third party material in this article are included in the article's Creative Commons license, unless indicated otherwise in a credit line to the material. If material is not included in the article's Creative Commons license and your intended use is not permitted by statutory regulation or exceeds the permitted use, you will need to obtain permission directly from the copyright holder. To view a copy of this license, visit http://creativecommons.org/licenses/by/4.0/.

(c) The Author(s) 2020 